\title{
Simulación, Implementación y Pruebas de un Sistema de Compensación en Corriente Directa basado en Ultra Capacitores, para Sistemas Ferroviarios
}

\author{
Mauricio Figueroa $^{(1)}$, Andrés E. Diez ${ }^{(1)}$, Luis E. Castrillón ${ }^{(2)}$ y Edison Manrique ${ }^{(2)}$ \\ (1) Universidad Pontificia Bolivariana, Facultad Ing. Eléctrica y Electrónica, Bloque 11, Medellín-Colombia \\ (2) Empresa de Transporte Masivo del Valle de Aburrá Limitada, Bello-Colombia \\ (e-mail: mauricio.figueroa@upb.edu.co; andres.diez@upb.edu.co; LCastrillon@metrodemedellin.gov.co; \\ EManrique@metrodemedellin.gov.co)
}

Recibido May. 17, 2016; Aceptado Jul. 14, 2016; Versión final Ago. 10, 2016, Publicado Feb. 2017

\begin{abstract}
Resumen
Se estudia la aplicación de sistemas de compensación basados en ultra capacitores en el sistema de potencia del Metro de Medellín. Los sistemas de compensación basados en ultra capacitores permiten mejorar la eficiencia energética de sistemas ferroviarios, al evitar que parte de la energía regenerada en frenados sea disipada en forma de calor, logrando al mismo tiempo estabilizar la tensión de alimentación. Con la implementación de estos sistemas también se aprovecha mejor la infraestructura de alimentación eléctrica, permitiendo expansiones de la línea y/o incremento de la flota del sistema, con menores inversiones. Para la aplicación estudiada se efectuó un diagnóstico del sistema de alimentación para evaluar los requerimientos y beneficios de implementar un compensador. El diseño del sistema compensador fue asistido por computador, usando un modelo digital que se describe en este trabajo. Finalmente se presentan los resultados obtenidos en operación real del dispositivo.
\end{abstract}

Palabras clave: frenado regenerativo, ultra capacitor, convertidor dc-dc, compensación de voltaje, sistema de transporte eléctrico

\section{Simulation and Implementation of a Direct Current Compensation System based on Ultra Capacitors for Railway Systems}

\begin{abstract}
The application of compensation systems based on ultra-capacitors in the power system of the Metro in Medellin is studied. Compensation systems based on ultra-capacitors allow improving energy efficiency of railway systems, by reducing the dissipated energy as heat from the regenerative braking, while contributing at the same to stabilize the voltage. Consequently, the implementation of these systems brings benefits in terms of usage of infrastructure, allowing either line or fleet expansion with less investment. For the application, the feed system was evaluated to determine the requirements and potential benefits of implementing a compensator. Compensator design was done with simulation assistance using a digital model described in this work. Finally, the results of the real operation of the device are presented.
\end{abstract}

Keywords: regenerative braking, ultra-capacitor, dc-dc converter, voltage compensation, electric transportation system 


\section{INTRODUCCIÓN}

En términos energéticos, la generación de energía eléctrica a partir de la energía cinética del tren (frenado regenerativo), permite una disminución del aporte del sistema a los vehículos, en virtud de que parte de la energía consumida al traccionar, es susceptible a ser recuperada (Lee et al, 2008). En el esquema actual de operación del Metro de Medellín, la mayor parte de esta energía regenerada se aprovecha por otro tren que se encuentre traccionando en la misma sección eléctrica del tren que frena. Cuando no hay tren consumidor, la tensión se eleva hasta que es necesaria la disipación en forma de calor en la resistencia de frenado. Esta última condición suele ser frecuente, por lo que una buena parte de la energía generada no es aprovechada sino convertida en calor no útil. En el caso del Metro de Medellín se estima que la energía disipada en calor es de 1,3 GWh al año (un 2,4 \% de la energía de tracción) (Lee et al., 2013). En la red eléctrica de Colombia, aunque predominantemente hidráulica, las emisiones de gases de efecto invernadero asociadas a la producción de la electricidad que se disipa en calor en los trenes, corresponde 130 TCO2 equivalentes al año.

Cuando un tren está frenando y existe consumo de energía por parte de trenes cercanos, de manera que la energía regenerada puede ser retornada al sistema de alimentación, se dice que la red está "receptiva" ocurriendo un fenómeno de compensación energética, que permite estabilizar la tensión, contribuyendo a que no se superen los límites operativos. Cuando se presenta la imposibilidad de retornar energía al sistema, por la ausencia de efecto de compensación, se considera que la red es no receptiva (Killer et al., 2012).

En general, cualquier maniobra de una carga produce un cambio de tensión en un sistema eléctrico, y esta fluctuación es proporcional a la potencia que es conectada o desconectada, y a la impedancia de la fuente en el punto de conexión. Los trenes se comportan como cargas de demanda fluctuante que tienden a provocar fluctuaciones de tensión de mayor magnitud, conforme se van alejando de las subestaciones de tracción, al aumentar la impedancia del sistema. El principal limitante para la extensión de una sección de alimentación, y por tanto del sistema de transporte, corresponde a la regulación de tensión. Esta restricción de longitud de expansión determina la distancia que debe haber entre las diferentes subestaciones de tracción que alimentan el sistema, y así mismo, define la distancia máxima de la última estación de pasajeros en el punto más alejado del sistema. Muchas veces, aunque las subestaciones tengan la capacidad de aportar la energía para una operación más lejana de los vehículos, esta distancia queda restringida por el cumplimiento de los niveles operativos de la tensión, que determinan el rango de operación segura de los vehículos (Capasso et al., 2008).

En la actualidad gracias al desarrollo de dispositivos de almacenamiento de energía en conjunto con convertidores basados en electrónica de potencia, es posible el desarrollo de equipos de compensación que permiten mejorar la receptividad de la red, con los beneficios subsecuentes: Mejora en la estabilidad de tensión: consecuencia natural del efecto de compensación (Erickson y Maksimović, 2001). Ahorro de energía: la energía no se disipa en resistencias, sino que es reutilizada para la tracción. Mejor utilización de la infraestructura del sistema de alimentación: al darse un aplanamiento en la curva de demanda y una mejora en la regulación de voltaje, la cantidad de trenes que se pueden alimentar con un mismo sistema se incrementa, y eventualmente, ante una expansión, la distancia de operación podría aumentarse (Battistelli et al., 2012). Este artículo trata sobre el diseño de un sistema de compensación estacionario para el Metro de Medellín, presentando el procedimiento para el dimensionamiento y ubicación del mismo, y el desempeño del prototipo que ha sido instalado. Para esto se parte de un estudio del comportamiento de la tensión del sistema, la caracterización de los trenes como cargas fluctuantes, y la modelación del sistema, comparando la operación actual (usada para validar el modelo), con la operación compensada, bajo diversas condiciones operativas (Barrero et al., 2008).

El uso de convertidores cd-cd es común en aplicaciones de almacenamiento de energía (ESS) por su alta eficiencia y su capacidad de actuar como controlador. En principio para esta aplicación se propone la combinación de dos tipos de convertidores cd-cd (Szenasy, 2008). El convertidor Buck se encarga de reducir la tensión de entrada, $V_{\text {in }}$, para cargar los ultra capacitores cuando el Metro está frenando; mientras que el convertidor Boost trabaja en el arranque elevando el voltaje del ultra capacitor, $\mathrm{V}_{\text {out, }}$ para alimentar la catenaria, y transferir la energía a un tren que pueda consumirla (Lee et al., 2008; Lee et al., 2013). En cuanto a la disponibilidad comercial de convertidores de cd-cd para controlar el banco de ultra capacitores, puede decirse que es limitada en los niveles de potencia, tensión y energía, que requieren los sistemas de tracción. Así mismo las restricciones de mercado y la especificidad de cada aplicación hacen que su costo sea una barrera. Sin embargo, al tener una topología simple y estar constituidos por relativamente pocos componentes, los convertidores de esta naturaleza constituyen un equipo con gran oportunidad de desarrollo local. 


\section{UBICACIÓN Y DIMENSIONAMIENTO}

Se parte de la inspección de la topología del sistema de suministro de energía eléctrica del sistema del Metro de Medellín. Cada sección de línea del Metro de Medellín tiene una alimentación en ca. de 13,8kV, con rectificación a $1,5 \mathrm{kV}$ de cd., que para el caso de estudio alimentan 2 líneas aéreas de contacto (ascendente y descendente) de 4,5 km de longitud por línea entre subestaciones de tracción. El esquema se puede observar en la Figura 1. El compensador se instala en al final de la sección de línea, el cual es el punto más alejado del sistema de alimentación y por tanto el más fluctuante en cuanto a los niveles de tensión se refiere, se identifica la estación de pasajeros Niquía como punto más fluctuante del sistema Metro de Medellín.

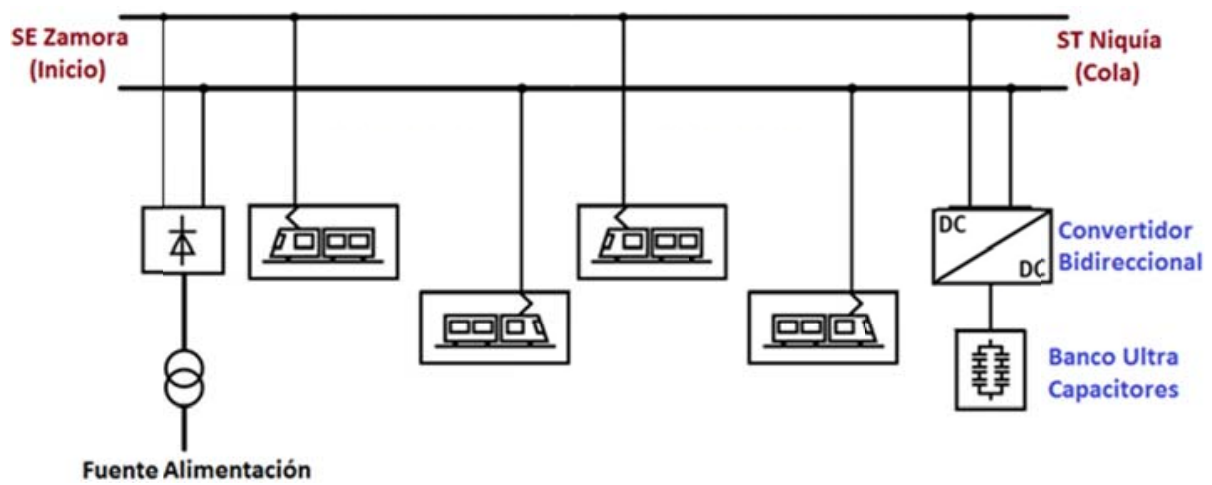

Fig. 1. Esquema sección de alimentación Metro de Medellín con Sistema de compensación usando ultra capacitores

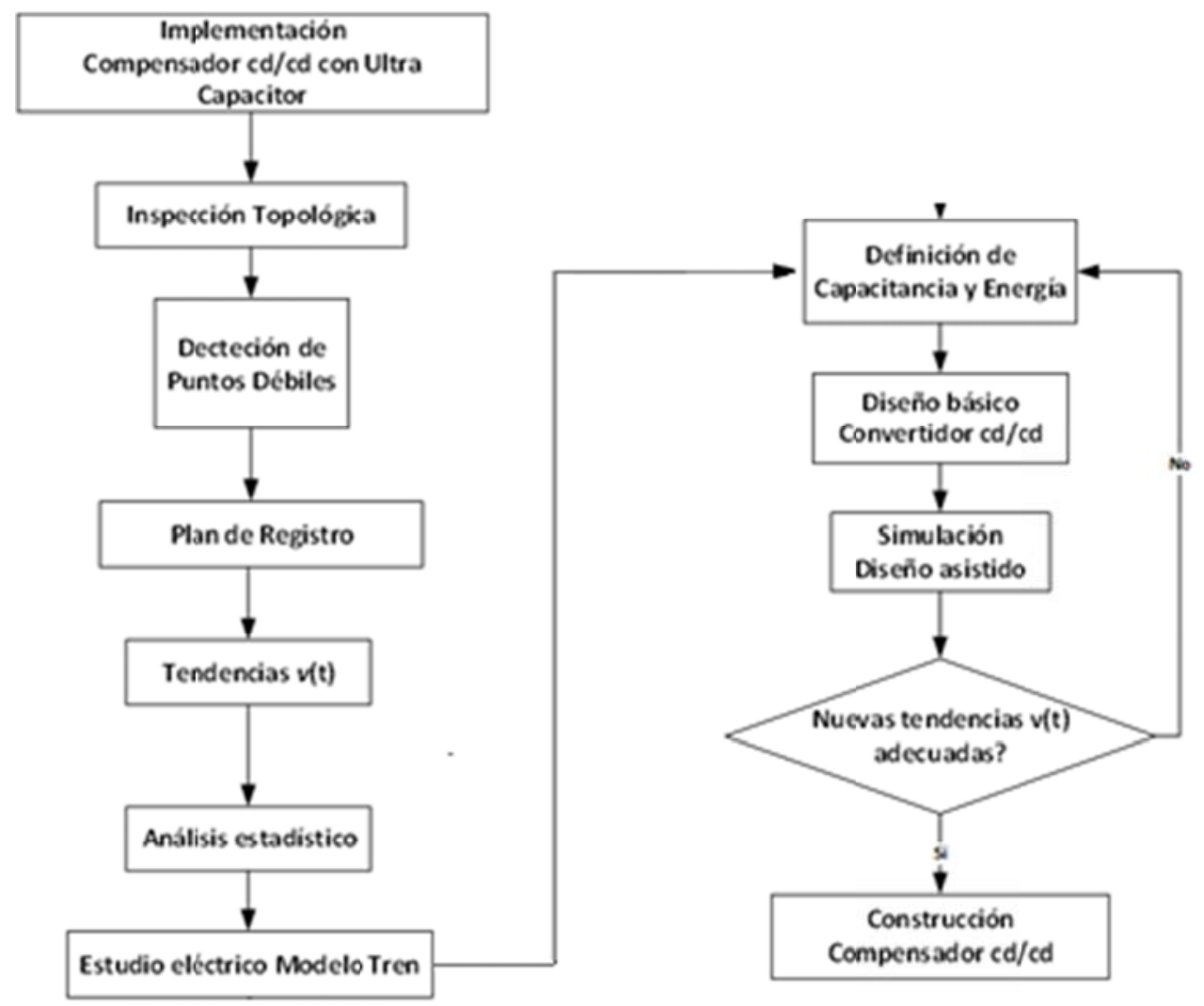

Fig. 2. Flujograma del estudio

El flujograma de la Figura 2, muestra la metodología que se sigue para establecer el estado y las necesidades del sistema de alimentación del Metro de Medellín; luego se procede a realizar un análisis estadístico complementario donde se establece la periodicidad de las fluctuaciones de tensión sobre la línea de contacto y determinar los causales de las mismas (típicamente el arranque de trenes) (García, 2010). 
Esta metodología establece que se debe realizar una simulación asistida por computador del modelo del tren sobre la línea, para lo cual se ha utilizado el PSCAD como herramienta computacional. Posteriormente se validan los resultados de la simulación sin compensador contra el sistema real, para constatar la veracidad de la simulación y procediendo finalmente con la implementación física del compensador como se ve en la Figura 3.

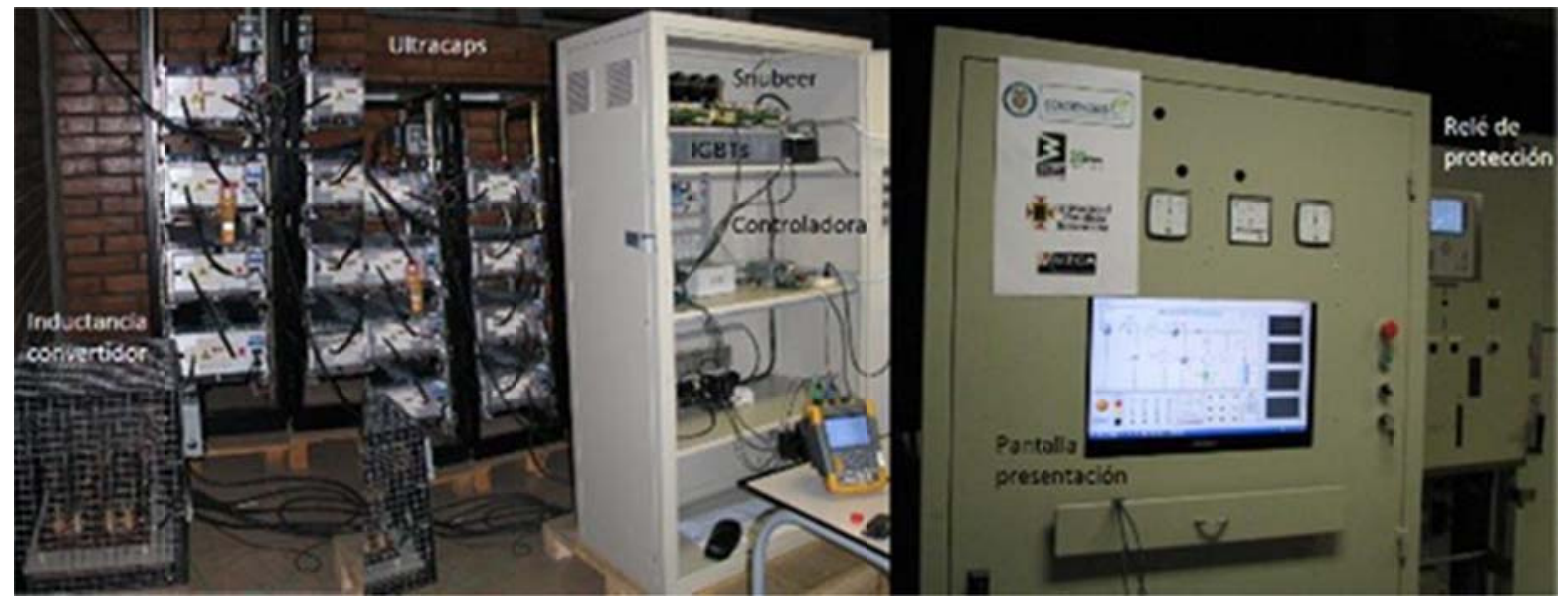

Fig. 3. Prototipo construido, convertidor a la izquierda y banco de Ultracapacitores a la derecha

El convertidor posee un sistema de control PI para limitar la tensión de salida, configurado dentro de los límites Vmáx $=1800 \mathrm{~V}$ y Vmín=1050 V y para la ganancia integral se añadió un bloque anti lazo. El convertidor posee también posee un controlador on-off para mantener la tensión del ultra capacitor dentro de los valores deseados. Cuando la tensión alcanza el límite inferior, el sistema deshabilita el convertidor Boost para mantener el flujo de carga del convertidor hacia el bus de cd y se mantiene deshabilitado hasta que la tensión alcanza un valor fijo que puede ser establecido en el punto medio entre las tensiones máximas y mínimas permitidas (Villegas et al., 2015). La tabla 1 muestra los parámetros principales del compensador.

Tabla 1. Especificación técnica general del convertidor cd-cd bidireccional (Buck - Boost)

\begin{tabular}{ccc}
\hline Parámetro & Valor & Comentario \\
\hline Voltaje & $1500 \mathrm{~V}$ & Voltaje nominal del convertidor \\
Corriente & $160 \mathrm{~A} / 240 \mathrm{~A}$ & Corriente máxima de operación del convertidor Buck/Boost \\
Potencia & $240 \mathrm{~kW}$ & Potencia máxima del sistema de compensación \\
Frecuencia & $3 \mathrm{kHz}$ & Frecuencia de operación del convertidor \\
\hline
\end{tabular}

\section{ANÁLISIS DE TENSIONES DE LÍNEA}

El Sistema Metro de Medellín, como los sistemas normalizados de $1500 \mathrm{~V}$ está diseñado para fluctuar en una banda $+20 \%$ y $-30 \%$ del valor nominal (Vmáx $=1800$ V, Vmín=1050 V). Cuando se alcanza la tensión de $1800 \mathrm{~V}$ efectuando frenado regenerativo, los trenes pasan a frenado disipativo, de forma que el exceso de energía se convierte en calor en las resistencias de frenado.

La Figura 4 muestra el perfil de tensión en línea de alimentación siendo la curva superior la correspondiente a la tensión en la estación de pasajeros más alejada del sistema y en la curva inferior, la tensión en la última subestación de tracción del sistema. Para efectos de fácil visualización, al perfil de tensión en la subestación de tracción (curva inferior) se le han restado $1000 \mathrm{~V}$, facilitando la comparación de las curvas en cada instante.

Se observa en el nodo de la subestación de tracción una diferencia de tensión, entre los valores máximo y mínimo de $844,4 \mathrm{~V}$, mientras que en la estación final (Niquía) del sistema esta diferencia es de 511,8 V, siendo evidente la diferencia en amplitud de las bandas de oscilación que tiene el voltaje en las dos estaciones. 


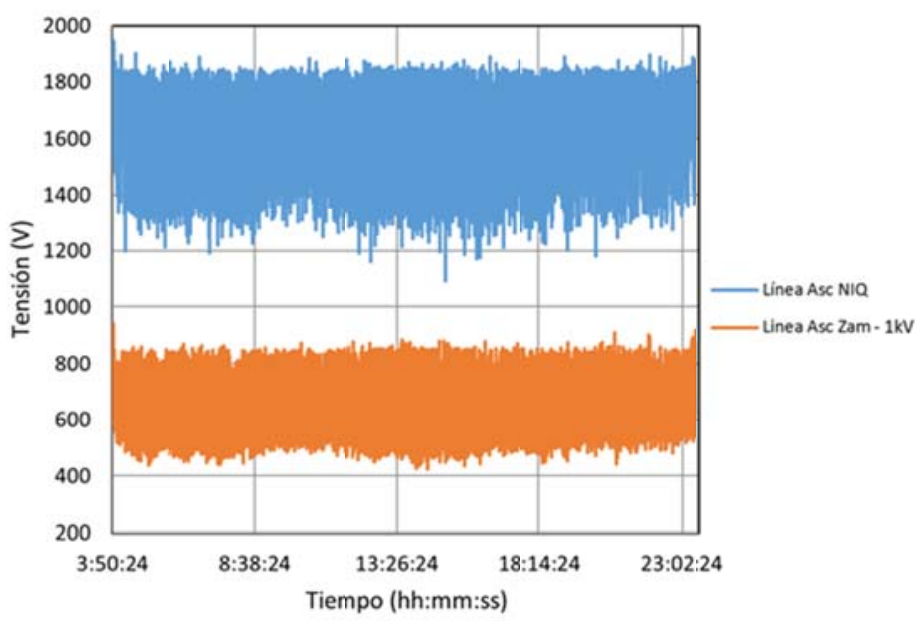

Fig. 4. Tensión de línea ascendente (Curva superior Voltaje en Niquía, la inferior es tensión en Acevedo menos mil voltios)

\section{ANÁLISIS ESTADÍSTICO}

Para poder establecer con precisión el valor del compensador necesario para reducir las fluctuaciones de tensión en el extremo del sistema, es necesario establecer el umbral para el cual se presentan la mayoría de las fluctuaciones y la periodicidad de las mismas. Se procede a obtener un histograma de la tensión durante el período de muestreo. En el punto más débil (ver Figura 5) se observa que el $10 \%$ de los datos registrados se encuentran por encima de $1800 \mathrm{~V}$, y el $20 \%$ de los datos por debajo de $1450 \mathrm{~V}$. Esto implica que un compensador que se ajuste para operar en la banda entre las tensiones $1450 \mathrm{~V}$ y $1800 \mathrm{~V}$, operaria el $30 \%$ tiempo.

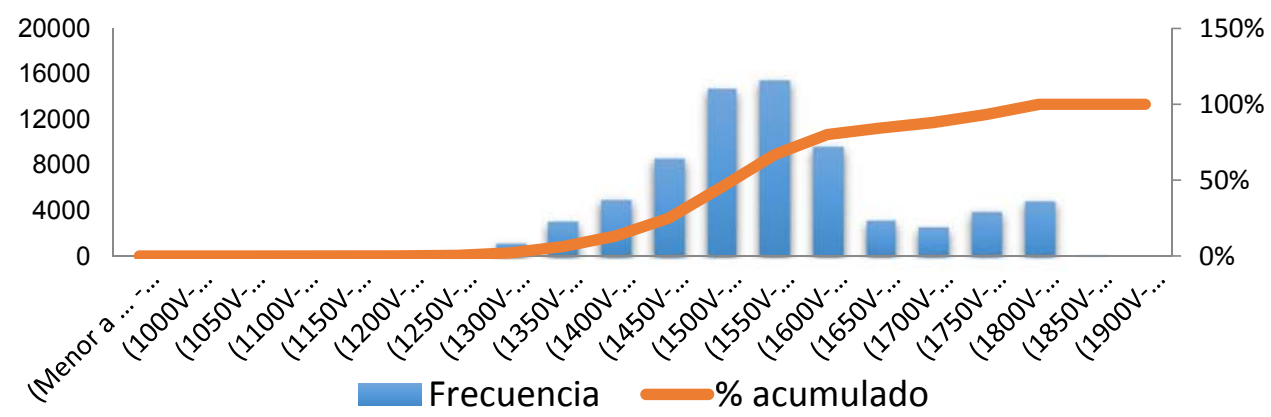

Fig. 5. Histograma de Frecuencia Línea Ascendente en la cola del sistema (1 día de muestreo)

Esto permite determinar de manera precisa el valor del compensador, así de esta manera minimizar inversiones innecesarias o mal calculadas para la implementación de estos sistemas de compensación.

\section{SELECCIÓN DEL TAMAÑO DE LA COMPENSACIÓN}

Para la simulación se consideraron condiciones estándar de aceleración hasta llegar a la velocidad máxima $(80 \mathrm{kmh})$ y desaceleración hasta llegar al paro total de 1 tren con carga promedio de pasajeros $(150 \mathrm{~T})$. Con base en los estudios anteriores desarrollados por la Universidad y el Metro (Killer et al., 2012), se puede considerar que la energía potencialmente recuperable $\left(E_{r}\right)$ es el $40 \%$ de la energía total cinética, que es lo que se almacenaría en el banco de condensadores, ver ecuación 1 :

$E_{r} \approx 4.1 k W h \approx \frac{1}{2} m v^{2} 0.4 \approx \frac{1}{2} c V^{2}$

Donde $c$ es la capacitancia y $\mathrm{V}$ la tensión del condensador, $m$ la masa del tren y $v$ su velocidad. El factor de 0.4 usado como eficiencia de recuperación, fue obtenido después de descontar a la energía cinética, las pérdidas por arrastre aerodinámico, fricción, caja reductora y las pérdidas eléctricas en el sistema de tracción. La condición ideal de almacenamiento se presenta cuando no hay un tren consumidor cercano, sin embargo, siempre es preferible que se dé la transferencia de tren a tren, sin que interceda el compensador. 
En los días festivos es común esta condición, que sirve para establecer el mayor tamaño práctico del compensador. Por el estudio del desempeño de los convertidores cd-cd (Teymourfar, 2011), se escoge una tensión de operación de $1000 \mathrm{~V}$. El banco estimado de ultra capacitor necesario para almacenar esa cantidad de energía en la SEU es formado por una conexión serie de 8 módulos individuales Maxwell BMOD0063, cada uno con capacitancia de $63 \mathrm{~F}, 125 \mathrm{~V}$. El banco final queda con las características presentadas en la tabla 2 .

Tabla 2: Banco estimado

\begin{tabular}{lcl}
\hline Parámetro & Valor & Comentario \\
\hline Voltaje & $1000 \mathrm{~V}$ & Máximo voltaje del Banco de Ultra capacitores \\
Capacitancia & $7,87 \mathrm{~F}$ & Capacitancia Total del Banco de Ultra capacitores \\
Resistencia Interna & $48 \mathrm{~m} \Omega$ & Resistencia Total del Banco de Ultra capacitores \\
Corriente & $240 \mathrm{~A}$ & Corriente continua RMS máxima $\left(\Delta \mathrm{T}=40^{\circ} \mathrm{C}\right)$ \\
\hline
\end{tabular}

\section{SIMULACIÓN CONVERTIDOR EN PSCAD}

Debido a la complejidad de estos sistemas, la mejor manera de anticipar su desempeño es el uso de simulaciones digitales. Para esto se escogió el PSCAD, programa en el cual se implementó un modelo simplificado del sistema de alimentación, correspondiente al equivalente de Thevenin en la última subestación de tracción (Zamora), la línea de alimentación (catenaria), los trenes operativos y finalmente, el sistema de compensación (Killer et al., 2012). El primer paso fue validar que el sistema virtual, sin compensador, tuviera el mismo comportamiento que el sistema real, comparando los efectos de las maniobras de los trenes simulados, con registros reales. Se debe comprobar que las fluctuaciones causadas coincidan en profundidad, duración y frecuencia. Comprobado el modelo, se procede a estudiar el desempeño del sistema de compensación para verificar los parámetros de diseño, y la forma en que interactúa con el sistema bajo diferentes esquemas de despacho de trenes, desde un solo tren en la sección eléctrica (días festivos) hasta cuatro unidades (hora pico). Incluso se efectúan variaciones en los tiempos de arranques y paradas buscando posibles casos críticos. Como ilustración la Figura 9, se presentan las curvas de tensión del sistema, tensión del Ultra Capacitor y la corriente del compensador, en el caso de operación hora pico. Cuando la tensión en línea se eleva por encima del umbral programado, el ultra capacitor se carga a través del convertidor bidireccional que se encuentra funcionando en modo Buck, cuando la tensión en línea disminuye, el ultra capacitor regresa la energía almacenada, y el convertidor opera en modo Boost, esto se repite cada vez que un tren arranca o frena, momentos en los cuales se presentan las mayores fluctuaciones de tensión sobre la línea de alimentación; el algoritmo de control se describe de manera detallada en (Villegas et al., 2015).

A pesar que en la simulación el rango de operación del capacitor se logró que fuera entre $1000 \mathrm{~V}$ (Vmáx) y $200 \mathrm{~V}$ (Vmín), en la práctica este rango debió limitarse a un Vmáx de $800 \mathrm{~V}$ y un Vmín de $350 \mathrm{~V}$. Esta tensión mínima para evitar inestabilidad y alcanzar valores prohibitivos de pérdidas.

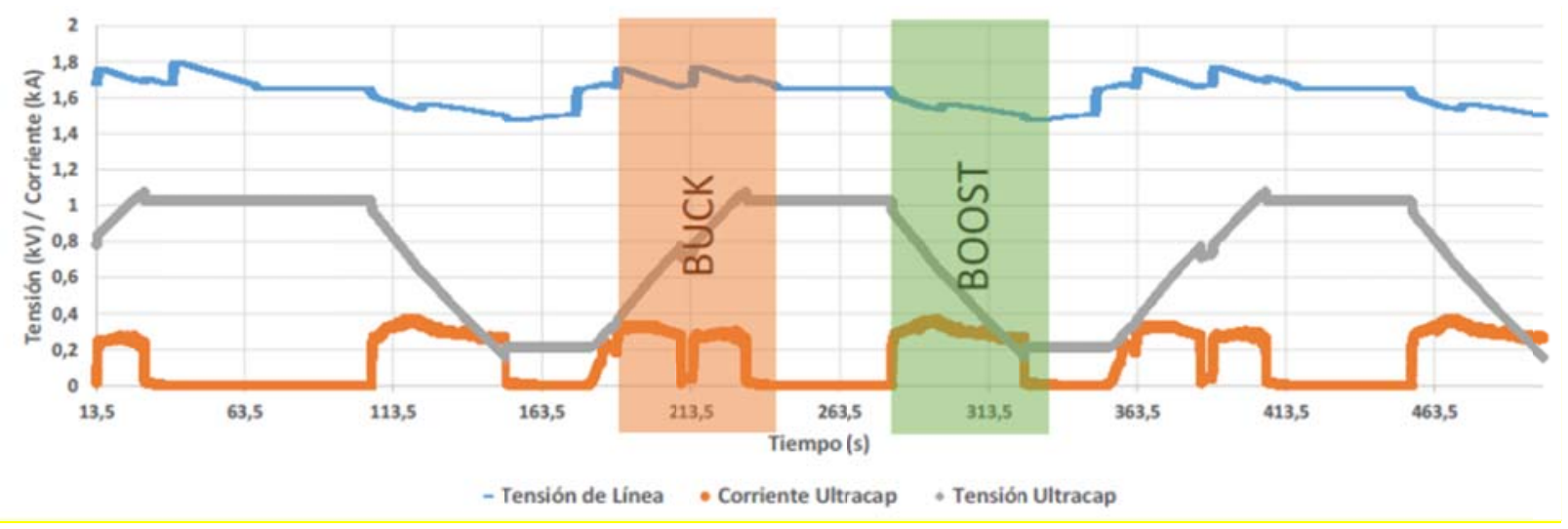

Fig.9. Funcionamiento en PSCAD del convertidor bidireccional Buck Boost de un tren con SEU de $1 \mathrm{kWh}$

La Figura 10 muestra el mismo caso de la figura anterior, pero ahora son datos reales medidos con el prototipo operando en la línea. Sin embargo, la corriente del equipo fue limitada a $160 \mathrm{~A}$ para operación Buck (toma de energía) y $90 \mathrm{~A}$ en Boost (retorno de energía). Se detectaron problemas de inestabilidad en el modo Boost al acercarse a los $100 \mathrm{~A}$, porque la operación pasaba a modo continuo, algo que no se recomienda. Como no pudieron ser superados los problemas del modo Boost, se optó por una operación 
asimétrica del convertidor, la cual se adecúo de buena manera al comportamiento del sistema. Para poder compensar la falta de velocidad de entrega de energía, se ajustó el umbral de voltaje de devolución al sistema para aportar durante más tiempo. El nuevo umbral escogido fue $1450 \mathrm{~V}$, valor inferior a la tensión nominal. El problema de inestabilidad no se pudo anticipar mediante la simulación en PSCAD, por la resolución de tiempo de la simulación, y el no tener en cuenta efectos derivados de las inductancias y capacitancias parásitas en el montaje real. Sin embargo, es notable la forma en que el modelo digital permitió anticipar el comportamiento real del compensador, en términos de volúmenes de energía, tiempos de operación y efecto sobre la tensión de red. Para la verificación de la operación estable en modos Buck y Boost se recomienda el uso de simulaciones digitales con resolución de microsegundos y pruebas con osciloscopio en sitio.

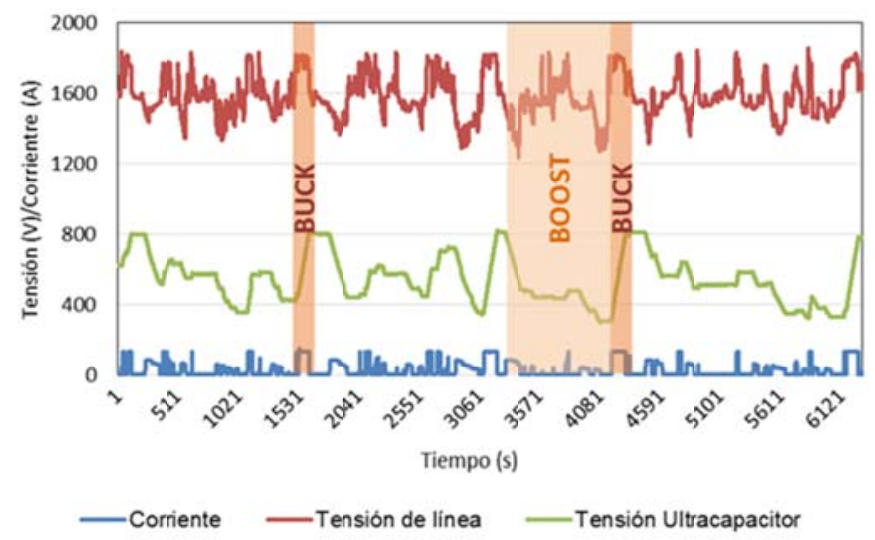

Fig. 10. Resultados prueba del prototipo del convertidor bidireccional.

En la Figura 11, permite comparar el voltaje del sistema compensado y sin compensar. Es evidente la manera en que la banda de oscilación es reducida, y como las excursiones de la tensión por debajo de los $1200 \mathrm{~V}$ son totalmente compensadas. El desempeño del equipo, a pesar de las limitaciones en corriente es satisfactorio.

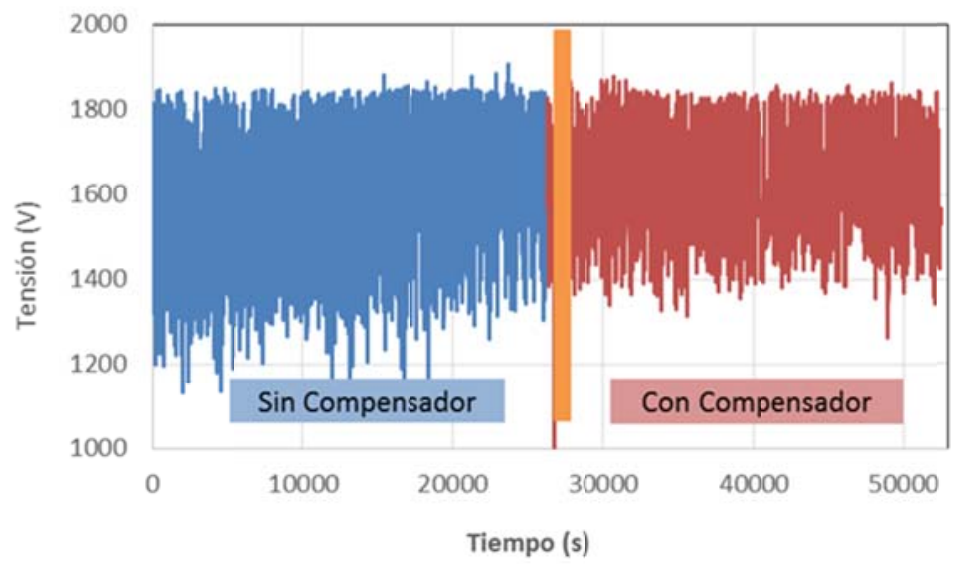

Fig. 11. Tensión en línea con y sin compensador.

En las pruebas se determina que la energía promedio por día recuperada es de $112 \mathrm{kWh}$, y el porcentaje de utilización del sistema es del $33 \%$, tal como lo anticipó el análisis estadístico y la simulación (ver Tabla 3).

Tabla 3: tiempo operación sistema

\begin{tabular}{ccccc} 
& Tiempo $[\mathrm{s}]$ & Tiempo $[\mathrm{m}]$ & Tiempo $[\mathrm{h}]$ & $\%$ \\
\hline Buck & 6634 & 110 & 1,843 & $10 \%$ \\
Boost & 15381 & 256 & 4,273 & $23 \%$ \\
\hline Total & 22015 & 366,917 & 6,115 & $33 \%$
\end{tabular}

Aunque el ahorro de energía no es suficiente (se debe valorar también el incremento de vida útil de los equipos) para pagar la inversión en un período de 10 años, un ejercicio financiero basado en la posibilidad de extender la línea de Metro $2 \mathrm{~km}$ adicionales hacia el norte, que compara la alternativa convencional de una subestación de tracción-SET-, contra la utilización de un compensador de 4 kWh (que permitiría 
mantener la operación dentro de los parámetros técnicos), muestra que los sistemas de compensación podrán, en el corto plazo, reducir los costos de inversión en infraestructura. El modelo financiero parte de una proyección del incremento anual del costo de energía del 3\%, de acuerdo a los contratos de largo plazo que posee el Metro de Medellín con el operador de red (EPM); se utilizó una tasa anual de descuento del $14 \%$. Se consideran una vida útil de 10 años para el ultra capacitor y de 30 años para el convertidor cd-cd y la SET. Los resultados muestran un VPN favorable al sistema de compensación, con un ahorro nominal anual de \$100.717.233 COP (unos \$33.000USD anuales), lo que le da viabilidad financiera al proyecto como lo muestra la tabla 4.

Tabla 4. Resultado del Modelo Financiero

\begin{tabular}{lcccc}
\hline \multicolumn{5}{c}{ VALOR PRESENTE NETO } \\
\hline \multicolumn{1}{c}{ Equipo } & Inversión & Costos & Ahorros energéticos & TOTAL \\
\hline Sistema de compensación SEU & $-\$ 6.214 .285 .833$ & $-\$ 1.263 .155$ & $\$ 377.560 .106$ & $-\$ 5.837 .988 .882$ \\
Subestación de tracción SET & $-\$ 8.846 .874 .332$ & $-\$ 12.631 .546$ & 0 & $-\$ 8.859 .505 .878$ \\
AHORROS EN VPN & $\$ 2.632 .588 .499$ & $\$ 11.368 .392$ & $\$ 377.560 .106$ & $\$ 3.021 .516 .996$ \\
\hline AHORRO NOMINAL ANUAL & & & & $\$ 100.717 .233$ \\
\hline
\end{tabular}

\section{CONCLUSIONES}

Los sistemas de compensación, como el desarrollado, aplicados a sistemas de tracción eléctrica, abren inmensas posibilidades para mejorar la estabilidad de tensión, ahorrar energía y un sobre aprovechamiento de la infraestructura del sistema de alimentación. Permiten un aplanamiento en la curva de demanda, mejor regulación de tensión y eventualmente, ante una expansión, la distancia de operación podría aumentarse. Así mismo, se incrementa la cantidad de trenes que se pueden alimentar con la infraestructura existente. El compensador no debe competir con la compensación natural que se da entre unidades que arrancan y frenan, por la propia dinámica de la operación. En este caso el umbral de captura de energía está fijado por la máxima tensión que obliga a disipación de calor, asegurando que no se compite con la compensación natural.

Estos sistemas pueden ser desarrollados en Latinoamérica a costos sensiblemente inferiores (relación 1:3) en comparación con desarrollos de compañías europeas y asiáticas. El esquema utilizado para el desarrollo involucró una compañía operadora (Metro de Medellín), una Universidad (UPB) y un socio tecnológico local para el montaje. Entidades gubernamentales han participado financiando parte del costo de las investigaciones (Colciencias, Ruta-N)

\section{AGRADECIMIENTOS}

Los autores agradecen el apoyo de las gerencias de Planeación, Operaciones y Mantenimiento del Metro de Medellín y a la coordinación de laboratorios de ingeniería eléctrica y electrónica de la Universidad Pontificia Bolivariana. A Ruta N por su apoyo mediante el proyecto titulado Redes Multimodales, código 111C-2015.

\section{REFERENCIAS}

Barrero, R., Tackoen, X. y Van Mierlo, J., Improving energy efficiency in public transport: stationary supercapacitor based energy storage systems for a metro-network. Vehicle Power and Propulsion Conference, 1-8.2009. Harbin-China, 3 al 5 Septiembre (2008)

Battistelli, L., Fantauzzi, M., lannuzzi, D. y Lauria, D., Energy management of electrified mass transit systems with Energy Storage devices, Int. Symp. Power Electron. Power Electron. Electr. Drives, Autom. Motion, 1172-1177, Junio (2012)

Capasso, A., Falvo, M. C., Grasselli, U., Lamedica, R., Bartoni, R., Francisi, M. y Maranzano, G., A planning study on power systems of metro-transit transportation system. 2008 Int. Symp. Power Electron. Electr. Drives, Autom. Motion, 1027-1032, Junio (2008)

Erickson R. W. y Maksimović D, Fundamentals of Power Electronics, Norwell (Mass.): Kluwer Acad. Publ, (2001)

García, X. del T. Ultracapacitor-based storage: Modelling, power conversion and energy considerations, ISIE (2010) IEEE, 2, 2493-2498, (2010) 
Killer, A., Armstorfer, A., Diez, A.E. y Biechl, H., Ultracapacitor assisted regenerative braking in metropolitan railway systems, doi: 10.1109/CITSS.2012.6336687, Intelligent Transportation Systems Symposium (CITSS), 1-6, 30 Agosto (2012)

Lee, H., Kim, G. y Lee, C., Development of ESS for Regenerative Energy of Electric Vehicle, Iccas, 316-321, (2008)

Lee, H.; Kim, G.; Lee, C.; Park, H., A Study on Saving Effects of Energy Regenerated from Electric Train, no. Iccas, 519-522, (2013)

Szenasy, I., Improvement the energy storage with ultracapacitor in metro railcar by modeling and simulation, IEEE Veh. Power Propuls. Conf., 2, 1, 1-5, Sepiembre (2008)

Teymourfar, R., Farivar, G., Iman-Eini, H. y Asaei, B., Optimal stationary super-capacitor energy storage system in a metro line. 2nd International Conference on Electric Power and Energy Conversion Systems (EPECS), 1-5, 15 al 17 Noviembre (2011)

Villegas, D., Diez, A.E., Restrepo, J.V., Munera, D., Bohórquez, A., Castrillon, L. y Manrique, E., Medium scale prototype of a regenerative braking system and voltage stabilizer in Power Electronics and Power Quality Applications (PEPQA), 2015 IEEE Workshop, 1-6, 2 al 4 Junio (2015) 
